\title{
Hyperon decay form factors in chiral perturbation theory*
}

\author{
André Lacour, Bastian Kubis \\ Helmholtz-Institut für Strahlen- und Kernphysik (Theorie), Universität Bonn \\ Nußallee 14-16, D-53115 Bonn, Germany \\ E-mail: lacour@itkp.uni-bonn.de, kubis@itkp.uni-bonn.de

\section{Ulf-G. Meißner} \\ Helmholtz-Institut für Strahlen- und Kernphysik (Theorie), Universität Bonn \\ Nußallee 14-16, D-53115 Bonn, Germany \\ and \\ Institut für Kernphysik (Theorie), Forschungszentrum Jülich, D-52425 Jülich, Germany \\ E-mail: meissner@itkp.uni-bonn.de
}

\begin{abstract}
We present a complete calculation of the SU(3)-breaking corrections to the hyperon vector form factors up to $\mathcal{O}\left(p^{4}\right)$ in covariant baryon chiral perturbation theory. Partial higher-order contributions are obtained, and we discuss chiral extrapolations of the vector form factor at zero momentum transfer. In addition we derive low-energy theorems for the subleading moments in hyperon decays, the weak Dirac radii and the weak anomalous magnetic moments, up to $\mathcal{O}\left(p^{4}\right)$.
\end{abstract}

KEYwords: Quark Masses and SM Parameters, Weak Decays, QCD, Chiral Lagrangians.

\footnotetext{
${ }^{*}$ Work supported in part by funds provided from the EU to the project "Study of Strongly Interacting Matter" under contract no. RII3-CT-2004-506078 and by the DFG (SFB/TR 16, "Subnuclear Structure of Matter"), and by the EU Contract No. MRTN-CT-2006-035482, "FLAVIAnet".
} 


\section{Contents}

1. Introduction 1

2. Vector form factors 3

3. Chiral Lagrangians 4

4. The Dirac form factor at zero momentum transfer 5

4.1 Heavy-baryon results up to order $p^{4} \quad 6$

$\begin{array}{lll}4.2 & \text { Order } p^{5} \text { corrections and numerical results } & 7\end{array}$

4.3 Chiral extrapolations 8

4.4 Further comments on the chiral expansion 11

5. Weak radii 11

6. Weak anomalous magnetic moments 12

7. Conclusions and outlook 14

A. Loop corrections for the weak Dirac radii $\quad 15$

B. Loop corrections for the weak anomalous magnetic moments 16

\section{Introduction}

Hyperon semileptonic decays are interesting for various reasons as they give information on the weak and the strong interactions in the light quark sector of QCD (for some recent experimental determinations, see e.g. Refs. [1-5]). The transition matrix elements are parameterized in terms of three vector current and three axial current form factors. Of these, the so-called vector form factor at zero momentum transfer, $f_{1}(0)$, plays a particular role. Hyperon decay data allow one to extract $\left|V_{u s} \cdot f_{1}(0)\right|^{2}$, where $V_{u s}$ is one entry of the Cabibbo-Kobayashi-Maskawa matrix. Deviations from SU(3) symmetry are expected to be very small because the Ademollo-Gatto theorem protects $f_{1}(0)$ from the leading $\mathrm{SU}(3)$ breaking corrections [6]. Therefore, precise calculations of the hadronic corrections to $f_{1}(0)$ appear feasible, resulting eventually in an accurate extraction of $V_{u s}$ from hyperon decays (for a recent analysis of SU(3) breaking effects in hyperon decays, see Ref. [7] and references therein).

In this paper, we will concentrate on the leading moments of the vector current form factors in semileptonic hyperon decays. There are two privileged frameworks for calculating 
the QCD corrections to these form factors, namely lattice QCD and chiral perturbation theory. First exploratory lattice studies are just becoming available, see Refs. [8-11]. The application of chiral perturbation theory to the semileptonic hyperon form factors has a longer history, see Refs. [12-15], with partly contradictory or incomplete results: Ref. [12] neglects the mass splitting in the baryon ground state octet, while Ref. [13] is erroneous with respect to the signs of certain contributions and misses some $1 / m$ corrections. Ref. [14] is purely confined to the leading-loop contributions. In Ref. [15], in addition the contributions of dynamical spin-3/2 decuplet intermediate states were considered in what is known as the small-scale expansion [16] generalization of chiral perturbation theory with baryons, which sometimes leads to an improved convergence behavior of the low-energy expansion (see e.g. Refs. [17-19]).

Except for the first of these studies, heavy-baryon chiral perturbation theory was utilized. Recently, a method was established to perform calculations in baryon chiral perturbation theory $(\mathrm{B} \chi \mathrm{PT})$ in a manifestly covariant way [20] (for a recent review discussing also different formulations of covariant $\mathrm{B} \chi \mathrm{PT}$ see Ref. [21]). It is therefore timely to revisit the calculation of the hyperon vector form factors in that framework. In what follows, we perform the full one-loop $\mathcal{O}\left(p^{4}\right)$ calculation in covariant $\mathrm{B} \chi \mathrm{PT}$ of hyperon decays, which may serve as a check of previous results [15] in a different regularization scheme, but in addition provides partial higher-order corrections useful for a study of the convergence behavior of the chiral series. In doing so, we revert to $\mathrm{B} \chi \mathrm{PT}$ without dynamical decuplet degrees of freedom, which in the light of surprisingly big effects found in Ref. [15] may be considered problematic. However, we want to concentrate on the resummation of higherorder loop effects and therefore defer an even more involved calculation of the decuplet effects in infrared regularization to a later study.

In addition to the already mentioned vector form factor at zero momentum transfer, we also calculate further observables such as weak radii and the weak anomalous magnetic moments. Those observables have become measurable nowadays $[2,3]$, and more results are expected from high-energy colliders in the future.

The paper is organized as follows. We define the vector form factors and explain their role in semileptonic hyperon decays in Sect. 2. In Sect. 3 we present the chiral Lagrangians necessary for our calculation and discuss the various low-energy constants. In Sect. 4 we present our results for the form factor $f_{1}$ at vanishing momentum transfer, confirming findings of Ref. [15], and discussing partial higher-order corrections. As the convergence behavior of $\mathrm{SU}(3) \mathrm{B} \chi \mathrm{PT}$ is known to be problematic, we investigate various chiral extrapolations in Sect. 4.3. In Sects. 5 and 6 we discuss the weak Dirac radii and the weak anomalous magnetic moments of semileptonic hyperon decays. The conclusions are given in Sect. 7. 


\section{Vector form factors}

The structure of ground state hyperon decays as probed by a charged strangeness-changing weak $\mathrm{SU}(3)$ vector current $V^{\mu}=V_{u s} \bar{u} \gamma^{\mu} s$ is parameterized in term of three form factors,

$$
\left\langle B^{\prime}\left(p_{2}\right)\left|V^{\mu}\right| B\left(p_{1}\right)\right\rangle=V_{u s} \bar{u}\left(p_{2}\right)\left[\gamma^{\mu} f_{1}^{B B^{\prime}}(t)+\frac{i \sigma^{\mu \nu} q_{\nu}}{m_{1}} f_{2}^{B B^{\prime}}(t)+\frac{q^{\mu}}{m_{1}} f_{3}^{B B^{\prime}}(t)\right] u\left(p_{1}\right),
$$

with the momentum transfer $q^{\mu}=p_{2}^{\mu}-p_{1}^{\mu}, t=q^{2}$, and $\sigma^{\mu \nu}=i\left[\gamma^{\mu}, \gamma^{\nu}\right] / 2 . m_{1}\left(m_{2}\right)$ is the mass of the initial (final) state baryon. $f_{1}$ is sometimes referred to as the vector form factor, $f_{2}$ as the weak magnetism form factor, and $f_{3}$ the induced scalar form factor. The expansion of these form factors at small momentum transfers defines slope parameters $\lambda_{i}$ or, in analogy to electromagnetic form factors, radii,

$$
f_{i}(t)=f_{i}(0)\left\{1+\frac{1}{6}\left\langle r_{i}^{2}\right\rangle t+\mathcal{O}\left(t^{2}\right)\right\}=f_{i}(0)\left\{1+\lambda_{i} \frac{t}{m_{1}^{2}}+\mathcal{O}\left(t^{2}\right)\right\},
$$

such that $\lambda_{i}=m_{1}^{2}\left\langle r_{i}^{2}\right\rangle / 6$.

We consider the following strangeness-changing $(s \rightarrow u)$ decays in the ground-state baryon octet

$$
\Lambda \rightarrow p \ell^{-} \bar{\nu}_{\ell}, \Sigma^{0} \rightarrow p \ell^{-} \bar{\nu}_{\ell}, \Sigma^{-} \rightarrow n \ell^{-} \bar{\nu}_{\ell}, \Xi^{-} \rightarrow \Lambda \ell^{-} \bar{\nu}_{\ell}, \Xi^{-} \rightarrow \Sigma^{0} \ell^{-} \bar{\nu}_{\ell}, \Xi^{0} \rightarrow \Sigma^{+} \ell^{-} \bar{\nu}_{\ell},
$$

where the lepton pair $\ell^{-} \bar{\nu}_{\ell}$ can be electronic or muonic.

Vector $(V)$ and axial vector $(A)$ current contributions do not interfere in the total decay rate $\Gamma=\Gamma_{A}+\Gamma_{V}$, and $\Gamma_{V}$ is related to the form factors Eq. (2.1) by [22]

$$
\begin{aligned}
\Gamma_{V}=G_{F}^{2}\left|V_{u s}\right|^{2} \frac{\Delta m^{5}}{60 \pi^{3}}\{ & {\left[1-\frac{3}{2} \beta+\frac{6}{7} \beta^{2}\left(1+\frac{1}{9} m_{1}^{2}\left\langle r_{1}^{2}\right\rangle\right)\right]\left|f_{1}(0)\right|^{2} } \\
& \left.+\frac{6}{7} \beta^{2}\left(\operatorname{Re} f_{1}(0) f_{2}(0)^{*}+\frac{2}{3}\left|f_{2}(0)\right|^{2}\right)+\mathcal{O}\left(\beta^{3}, m_{\ell}^{2}\right)\right\},
\end{aligned}
$$

where $\beta=\Delta m / m_{1}=\left(m_{1}-m_{2}\right) / m_{1}, G_{F}$ is the Fermi constant, and $m_{\ell}$ denotes the lepton mass, $\ell=e, \mu$. We note that the induced scalar form factor $f_{3}$ is suppressed by $m_{\ell}^{2}$ and can safely be neglected at least in the electron channel; we will not consider $f_{3}$ any further in this work. The expansion in the small quantity $\beta$ in Eq. (2.4) demonstrates that the decay width is dominated by $f_{1}(0)$, and that subleading contributions are given by the Dirac radius $\left\langle r_{1}^{2}\right\rangle$ as well as by the weak magnetism form factor at vanishing momentum transfer, $f_{2}(0)$. Both of these subleading moments will hence be discussed in the following.

In the $\mathrm{SU}(3)$ limit the vector form factors at zero momentum transfer $f_{1}(0)$ are fixed by the conservation of the $\mathrm{SU}(3)_{V}$ charge. The Ademollo-Gatto theorem [6] asserts that $\mathrm{SU}(3)$ breaking effects only start at second order in the symmetry breaking term $\left(m_{s}-\hat{m}\right)$,

$$
f_{1}(0)=f_{1}^{\mathrm{SU}(3)}(0)+\mathcal{O}\left(\left(m_{s}-\hat{m}\right)^{2}\right),
$$

with the average small quark mass $\hat{m}=\left(m_{u}+m_{d}\right) / 2 . \quad f_{1}^{\mathrm{SU}(3)}(0) \equiv g_{V}$ are the vector couplings in the symmetry limit, which read:

$$
g_{V}^{\Lambda p}=-\sqrt{\frac{3}{2}}, \quad g_{V}^{\Sigma^{0} p}=-\frac{1}{\sqrt{2}}, \quad g_{V}^{\Sigma^{-} n}=-1, \quad g_{V}^{\Xi^{-} \Lambda}=\sqrt{\frac{3}{2}}, \quad g_{V}^{\Xi^{-} \Sigma^{0}}=\frac{1}{\sqrt{2}}, \quad g_{V}^{\Xi^{0} \Sigma^{+}}=1
$$


Since isospin breaking effects are much smaller than SU(3) breaking effects $\left(m_{d}-m_{u} \ll\right.$ $\left.m_{s}-\hat{m}\right)$, we neglect the former. Isospin symmetry then relates the transitions $\Sigma^{0} \rightarrow p$ and $\Sigma^{-} \rightarrow n$, as well as $\Xi^{-} \rightarrow \Sigma^{0}$ and $\Xi^{0} \rightarrow \Sigma^{+}$in a trivial manner: when dividing by the overall vector charge $g_{V}$, the corresponding form factors are equal, hence the number of independent processes reduces from six to four.

\section{Chiral Lagrangians}

We will employ chiral perturbation theory $(\chi \mathrm{PT})[23-25]$ as the effective theory in the low-energy region of QCD (for a recent review see e.g. Ref. [26]).

The chiral effective pseudo-Goldstone boson Lagrangian to leading order is given by

$$
\mathcal{L}_{\phi}^{(2)}=\frac{F_{\pi}^{2}}{4}\left\langle u_{\mu} u^{\mu}+\chi^{+}\right\rangle
$$

where $u_{\mu}=i u^{\dagger} \nabla_{\mu} U u^{\dagger}, u^{2}=U$ collects the Goldstone boson fields in the usual manner, and $\chi^{+}=u \chi^{\dagger} u+u^{\dagger} \chi u^{\dagger}, \chi=2 B \operatorname{diag}\left(m_{u}, u_{d}, m_{s}\right)+\ldots$ incorporates the quark masses. As the notation suggests, we can identify the Lagrangian's normalization constant with the pion decay constant for the purpose of this study, $F_{\pi}=92.4 \mathrm{MeV}$.

For the effective meson-baryon Lagrangian we employ basis and notation of Refs. [27, 28] (see the related work in Ref. [29]). At leading order, it reads

$$
\mathcal{L}_{\phi B}^{(1)}=\left\langle\bar{B}\left(i \gamma^{\mu}\left[D_{\mu}, B\right]-m B\right)\right\rangle+\frac{D / F}{2}\left\langle\bar{B} \gamma^{\mu} \gamma_{5}\left[u_{\mu}, B\right]_{ \pm}\right\rangle
$$

where $B$ is the matrix of the ground state octet baryon fields, $m$ is the average octet mass and $D$ and $F$ are the axial vector coupling constants (strictly speaking, the parameters appearing in the Lagrangian refer to the chiral SU(3) limit). Their numerical values can be extracted from hyperon decays and obey the $\mathrm{SU}(2)$ constraint for the axial vector coupling $g_{A}=D+F=1.26$; we use $D=0.80, F=0.46$. The following terms from the baryonmeson Lagrangian at second order are needed to generate the baryon mass splittings at leading order, as well as the coupling of (traceless) vector currents:

$$
\begin{aligned}
\mathcal{L}_{\phi B}^{(2)}=b_{D / F} & \left\langle\bar{B}\left[\chi^{+}, B\right]_{ \pm}\right\rangle+i b_{5 / 6}\left\langle\bar{B} \sigma^{\mu \nu}\left[\left[u_{\mu}, u_{\nu}\right], B\right]_{\mp}\right\rangle \\
& +i b_{7}\left\langle\bar{B} u_{\mu}\right\rangle \sigma^{\mu \nu}\left\langle u_{\nu} B\right\rangle+b_{12 / 13}\left\langle\bar{B} \sigma^{\mu \nu}\left[F_{\mu \nu}^{+}, B\right]_{\mp}\right\rangle .
\end{aligned}
$$

We use the numerical values $b_{5}=0.23 \mathrm{GeV}^{-1}, b_{6}=0.62 \mathrm{GeV}^{-1}, b_{7}=0.68 \mathrm{GeV}^{-1}$ obtained from resonance saturation estimates [30,31]. To the order we consider here, the effects of $b_{D / F}$ can always be re-expressed in terms of the physical baryon masses, for which we employ $m_{N}=0.939 \mathrm{GeV}, m_{\Lambda}=1.116 \mathrm{GeV}, m_{\Sigma}=1.193 \mathrm{GeV}$, and $m_{\Xi}=1.318 \mathrm{GeV}$. In addition, we will occasionally refer to an average octet baryon mass $m=1.151 \mathrm{GeV}$. Finally, $b_{12 / 13}$ can at leading order be determined from the anomalous magnetic moments of proton and neutron.

Only two terms, entering the Dirac radii of the baryons, are needed from the third order Lagrangian,

$$
\mathcal{L}_{\phi B}^{(3)}=d_{51 / 52}\left\langle\bar{B} \gamma^{\mu}\left[\left[D^{\nu}, F_{\mu \nu}^{+}\right], B\right]_{\mp}\right\rangle
$$




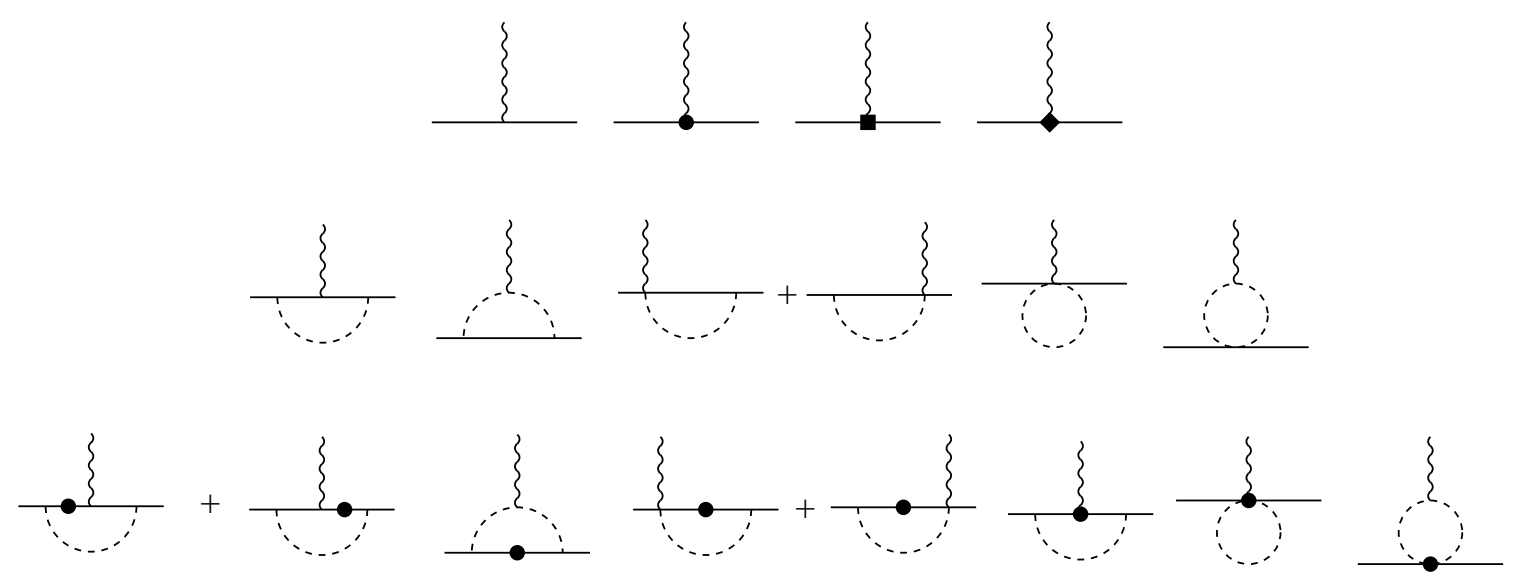

Figure 1: Feynman diagrams contributing to the vector current form factors up to fourth order. Solid, dashed, and wiggly lines refer to baryons, Goldstone bosons, and the weak vector source, respectively. Vertices denoted by a heavy dot/square/diamond refer to insertions from the second/third/fourth order chiral Lagrangian, respectively. Diagrams contributing via wave function renormalization only are not shown. Note that the masses appearing in the various propagators differ in the initial and final states and may also be different for the intermediate states.

$d_{51 / 52}$ can be determined from the Dirac (or electric) radii of the nucleons [31,32]. At fourth order seven couplings proportional to a quark mass insertion contributing to the anomalous magnetic moments are of relevance:

$$
\begin{aligned}
\mathcal{L}_{\phi B}^{(4)}= & \alpha_{1 / 2}\left\langle\bar{B} \sigma^{\mu \nu}\left(\left[\left[F_{\mu \nu}^{+}, B\right], \chi^{+}\right]_{\mp}+\left[F_{\mu \nu}^{+},\left[B, \chi^{+}\right]_{\mp}\right]\right)\right\rangle \\
& +\alpha_{3 / 4}\left\langle\bar{B} \sigma^{\mu \nu}\left(\left[\left\{F_{\mu \nu}^{+}, B\right\}, \chi^{+}\right]_{\mp}+\left\{F_{\mu \nu}^{+},\left[B, \chi^{+}\right]_{\mp}\right\}\right)\right\rangle \\
& +\alpha_{5}\left\langle\bar{B} \sigma^{\mu \nu} B\right\rangle\left\langle F_{\mu \nu}^{+} \chi^{+}\right\rangle+\alpha_{6 / 7}\left\langle\bar{B} \sigma^{\mu \nu}\left[F_{\mu \nu}^{+}, B\right]_{\mp}\right\rangle\left\langle\chi^{+}\right\rangle .
\end{aligned}
$$

The term proportional to $\alpha_{5}$ vanishes for off-diagonal currents, and hence for weak decay matrix elements, while $\alpha_{6 / 7}$ account for a quark mass renormalization of the magnetic couplings $b_{12 / 13}$. The operators scaling with $\alpha_{1-5}$ incorporate explicit breaking of $\mathrm{SU}(3)$ symmetry in the anomalous magnetic moments and have to be fitted to the baryon octet's anomalous magnetic moments $[30,31] . \quad \mathcal{L}_{\phi B}^{(4)}$ also contains two additional counterterms contributing to the magnetic (Pauli) radii [31], which however we will not consider here.

\section{The Dirac form factor at zero momentum transfer}

We will calculate the loop diagrams in a manifestly covariant form, using infrared regularization [20]; for the diagrams that are to be considered, see Fig. 1.

In comparison to a heavy-baryon calculation to subleading one-loop order [15], there are far fewer diagrams to be considered, as all the $1 / m$ corrections and baryon mass splittings in the baryon propagators are automatically resummed to all orders. On the other hand, the closed forms for the full loop results are much more involved and cannot be displayed here completely. A re-expansion of the covariant loop diagrams in strict chiral power counting 
however reproduces the heavy-baryon results and leads to simplified expressions that are useful for comparison with the literature.

The Ademollo-Gatto theorem results in the absence of local contributions up to fourth chiral order, therefore our analysis up that order is free from low-energy constants. We will parameterize the expanded corrections in analogy to Ref. [15],

$$
f_{1}(0)=g_{V}\left[1+\delta^{(2)}+\left(\delta^{(3,1 / m)}+\delta^{(3, \delta m)}\right)+\delta^{(4 *)}+\ldots\right]
$$

$\delta^{(2)}$ is the leading $\mathrm{SU}(3)$-breaking loop correction of order $p^{3}$. The corrections of order $p^{4}$ are divided into two classes, pure $1 / m$ recoil corrections $\delta^{(3,1 / m)}$ and terms proportional to the baryon mass splitting denoted by $\delta^{(3, \delta m)}$. As an indicator for the size of higherorder terms, we can also extract partial (i.e. incomplete) corrections of order $p^{5}$ from the covariant amplitudes, which we denote by $\delta^{(4 *)}$ (the asterisk serving as a reminder that there are additional, e.g. two-loop, corrections at that order).

\subsection{Heavy-baryon results up to order $p^{4}$}

Both self-energy like diagrams and tadpoles contribute to $f_{1}(0)$ at this order, the former scaling with the axial couplings $D$ and $F$ squared, the latter coming with completely fixed coefficients. The results read:

$$
\delta_{B B^{\prime}}^{(2)}+\delta_{B B^{\prime}}^{(3,1 / m)}=3\left(H_{\pi K}^{(1)}+H_{\eta K}^{(1)}\right)+\gamma_{B B^{\prime}}^{\pi}\left(H_{\pi K}^{(1)}+H_{\pi K}^{(2)}\right)+\gamma_{B B^{\prime}}^{\eta}\left(H_{\eta K}^{(1)}+H_{\eta K}^{(2)}\right)
$$

where the coefficients $\gamma_{B B^{\prime}}^{\pi / \eta}$ are shown in Table 1 , and the functions $H_{a b}^{(1)}, H_{a b}^{(2)}$ are given by

$$
\begin{aligned}
& H_{a b}^{(1)}=\frac{1}{\left(8 \pi F_{\pi}\right)^{2}}\left\{\frac{M_{a}^{2} M_{b}^{2}}{M_{b}^{2}-M_{a}^{2}} \log \frac{M_{b}}{M_{a}}-\frac{1}{4}\left(M_{a}^{2}+M_{b}^{2}\right)\right\} \\
& H_{a b}^{(2)}=\frac{\pi}{6 m\left(8 \pi F_{\pi}\right)^{2}} \frac{\left(M_{b}-M_{a}\right)^{2}}{M_{a}+M_{b}}\left(M_{a}^{2}+3 M_{a} M_{b}+M_{b}^{2}\right) .
\end{aligned}
$$

The corrections Eqs. (4.3), (4.4) satisfy the Ademollo-Gatto theorem, and have been given before in the literature $[12,14,15]$. As already stated in Refs. [33,34], the quadratic symmetry breaking term $\left(m_{s}-\hat{m}\right)^{2}$ comes with coefficients that scale with inverse powers of the quark masses, therefore allowing for (non-analytic) symmetrybreaking corrections at lower orders

\begin{tabular}{|c|cc|}
\hline$B \rightarrow B^{\prime}$ & $\gamma_{B B^{\prime}}^{\pi}$ & $\gamma_{B B^{\prime}}^{\eta}$ \\
\hline$\Lambda \rightarrow N$ & $9 D^{2}+6 D F+9 F^{2}$ & $(D+3 F)^{2}$ \\
$\Sigma \rightarrow N$ & $D^{2}-18 D F+9 F^{2}$ & $9(D-F)^{2}$ \\
$\Xi \rightarrow \Lambda$ & $9 D^{2}-6 D F+9 F^{2}$ & $(D-3 F)^{2}$ \\
$\Xi \rightarrow \Sigma$ & $D^{2}+18 D F+9 F^{2}$ & $9(D+F)^{2}$ \\
\hline
\end{tabular}

Table 1: Coefficients for Eq. (4.2). than what local (analytic) terms can provide. 
The baryon mass splitting corrections are somewhat more complicated, but can still be brought into a rather compact form,

$$
\begin{aligned}
\delta_{\Lambda N}^{(3, \delta m)}= & (D+F)(D+3 F) H_{K \pi}^{\Lambda N}\left(m_{N}\right)-\frac{1}{3}\left(D^{2}-9 F^{2}\right) H_{K \eta}^{\Lambda N}\left(m_{N}\right) \\
& +\frac{2}{3} D(D+3 F) H_{\eta K}^{\Lambda N}\left(m_{\Lambda}\right)+2 D(D-F) H_{\pi K}^{\Lambda N}\left(m_{\Sigma}\right), \\
\delta_{\Sigma N}^{(3, \delta m)}= & \left.D^{2}-F^{2}\right) H_{K \pi}^{\Sigma N}\left(m_{N}\right)+(D-F)(D-3 F) H_{K \eta}^{\Sigma N}\left(m_{N}\right) \\
& -\frac{2}{3} D(D+3 F) H_{\pi K}^{\Sigma N}\left(m_{\Lambda}\right)-4(D-F) F H_{\pi K}^{\Sigma N}\left(m_{\Sigma}\right)+2 D(D-F) H_{\eta K}^{\Sigma N}\left(m_{\Sigma}\right), \\
\delta_{\Xi \Lambda}^{(3, \delta m)}= & \frac{2}{3} D(D-3 F) H_{K \eta}^{\Xi \Lambda}\left(m_{\Lambda}\right)+(D-F)(D-3 F) H_{\pi K}^{\Xi \Lambda}\left(m_{\Xi}\right) \\
& -\frac{1}{3}\left(D^{2}-9 F^{2}\right) H_{\eta K}^{\Xi \Lambda}\left(m_{\Xi}\right)+2 D(D+F) H_{K \pi}^{\Xi \Lambda}\left(m_{\Sigma}\right), \\
\delta_{\Xi \Sigma}^{(3, \delta m)}= & -\frac{2}{3} D(D-3 F) H_{K \pi}^{\Xi \Sigma}\left(m_{\Lambda}\right)+\left(D^{2}-F^{2}\right) H_{\pi K}^{\Xi \Sigma}\left(m_{\Xi}\right)+4(D+F) F H_{K \pi}^{\Xi \Sigma}\left(m_{\Sigma}\right) \\
& +(D+F)(D+3 F) H_{\eta K}^{\Xi \Sigma}\left(m_{\Xi}\right)+2 D(D+F) H_{K \eta}^{\Xi \Sigma}\left(m_{\Sigma}\right),
\end{aligned}
$$

with

$$
\begin{aligned}
H_{a b}^{A B}(m)=\frac{\pi}{3\left(8 \pi F_{\pi}\right)^{2}} \frac{M_{b}-M_{a}}{\left(M_{a}+M_{b}\right)^{2}}\{ & \left(m_{B}-m_{A}\right)\left(M_{a}^{2}+3 M_{a} M_{b}+M_{b}^{2}\right) \\
& \left.+3\left(m-m_{A}\right) M_{b}^{2}-3\left(m-m_{B}\right) M_{a}^{2}\right\},
\end{aligned}
$$

satisfying the Ademollo-Gatto theorem. Eq. (4.5) agrees with the results given in Ref. [15].

\subsection{Order $p^{5}$ corrections and numerical results}

A useful benefit of the infrared regularization method is that a certain, well-defined subset of higher-order contributions, stemming from all possible $1 / m$ corrections (including, in our case, those due to the shift of the baryon masses away from their $\mathrm{SU}(3)$ symmetry limit) in the baryon propagators, are automatically resummed. In the case of the hyperon decay form factors, such higher-order corrections are far from being complete, but comprise a complete set of terms, namely those quadratic in the axial couplings $D$ and $F$. As a downside, these higher-order terms are in general not finite, and even after removing the infinities by hand, display some subleading renormalization scale dependence. We try to reflect the resulting inherent uncertainties by varying the scale between $M_{\rho}=0.770 \mathrm{GeV}$ and $m_{\Xi}=1.318 \mathrm{GeV}$ (with a central scale chosen at $1 \mathrm{GeV}$ ). Here we evaluate both the partial next-to-next-to-leading order $\delta^{(4 *)}$ and the completely resummed covariant loop results numerically. The analytic expressions are rather cumbersome and can be obtained in Ref. [35].

In Table 2 we give the numerical results for each contribution defined in Eq. (4.1) $\left(\delta^{(2)}\right.$, $\left.\delta^{(3,1 / m)}, \delta^{(3, \delta m)}, \delta^{(4 *)}\right)$ separately, the results summed up to given chiral orders $\mathcal{O}\left(p^{3}\right)-\mathcal{O}\left(p^{5}\right)$ $\left(\delta^{(2)}\right.$, Sum $^{(3)}$, Sum $\left.^{(4)}\right)$ and for the complete covariant expressions $(\mathrm{Cov})$. Bands for the variation of the renormalization scale as detailed above are given for $\delta^{(4 *)}, \operatorname{Sum}^{(4)}$, and Cov. The numerical results for $\delta^{(2)}, \delta^{(3,1 / m)}, \delta^{(3, \delta m)}$, and consequently Sum $^{(3)}$ agree with those of Ref. [15], as well as those for $\delta^{(2)}$ with Ref. [14]. 


\begin{tabular}{|c|cccc|ccc|}
\hline Channel & $\delta^{(2)}$ & $\delta^{(3,1 / m)}$ & $\delta^{(3, \delta m)}$ & $\delta^{(4 *)}$ & Sum $^{(3)}$ & Sum $^{(4)}$ & Cov \\
\hline$\Lambda \rightarrow N$ & -9.7 & +8.1 & +4.4 & $+3.8 \pm 3.2$ & +2.8 & $+6.6 \pm 3.2$ & $-5.7 \pm 2.1$ \\
$\Sigma \rightarrow N$ & +0.8 & -3.3 & +6.7 & $+2.3 \pm 0.6$ & +4.1 & $+6.4 \pm 0.6$ & $+2.8 \pm 0.2$ \\
$\Xi \rightarrow \Lambda$ & -6.3 & +4.4 & +6.3 & $+0.9 \pm 2.2$ & +4.4 & $+5.3 \pm 2.2$ & $-1.1 \pm 1.7$ \\
$\Xi \rightarrow \Sigma$ & -9.4 & +7.9 & +2.5 & $+3.5 \pm 2.6$ & +1.0 & $+4.4 \pm 2.6$ & $-5.6 \pm 1.6$ \\
\hline
\end{tabular}

Table 2: Numerical analysis of relative chiral corrections to the hyperon decay vector form factors. Values are given for each contribution separately and by chiral orders summed up $\left(\operatorname{Sum}^{(3)}=\right.$ $\delta^{(2)}+\delta^{(3,1 / m)}+\delta^{(3, \delta m)}$, Sum $\left.^{(4)}=S u m^{(3)}+\delta^{(4 *)}\right)$. The corrections are given in per cent $(\%)$.

Comparing with model approaches for the SU(3)-breaking corrections in $f_{1}(0)$, we note that quark models tend to yield small negative corrections of $-1.3 \%$ [36] or $-2.4 \%$ to $-2.5 \%$ [37] for all decay channels, while an analysis based on the $1 / N_{c}$ expansion of QCD [38] yields positive corrections throughout, ranging from $+2 \pm 2 \%$ for $\Lambda \rightarrow p$ to $+7 \pm 7 \%$ for $\Xi \rightarrow \Sigma$. While heavy-baryon $\chi \mathrm{PT}$ up to complete $\mathcal{O}\left(p^{4}\right)$ also favors positive corrections, the covariant resummation rather agrees in sign with the quark models in three out of four channels, see Table 2 .

We note that the $\Sigma \rightarrow N$ form factor is consistently found to receive positive corrections in Table 2, while there is a sum rule argument $[39,40]$ suggesting the opposite. The latter is based on the fact that positive contributions are due to states of exotic strangeness/isospin quantum numbers $S=-2, I=3 / 2$, and the assumption that the sum rule ought to be dominated by resonances. In sum rule language, the corrections calculated in $\mathrm{B} \chi \mathrm{PT}$ are due to non-resonant multi-particle intermediate states; as the effects of resonances are subsumed in counterterms, which only start to contribute at $\mathcal{O}\left(p^{5}\right)$ for the quantity at hand, the argument given in Refs. [39,40] leads to the expectation that such counterterm corrections are likely to reduce the positive correction in the $\Sigma \rightarrow N$ form factor.

It appears somewhat irritating that the sums of all terms up to (partial) $\mathcal{O}\left(p^{5}\right), \operatorname{Sum}^{(4)}$, are not at all close to the full covariant results, see Table 2, and even opposite in sign in three out of four channels: the chiral expansion seems to converge very slowly towards its own covariant resummation. This is a frequent problem in $\mathrm{SU}(3) \mathrm{B} \chi \mathrm{PT}$, see e.g. Refs. [4143]. We further investigate this issue in the following section by varying the quark masses.

\subsection{Chiral extrapolations}

In order to investigate the convergence behavior of the chiral corrections to $f_{1}(0)$ and to test the consistency of our method, we wish to extrapolate the quark masses towards the chiral limit and see if convergence in the spirit of chiral power counting is retrieved for smaller masses.

To investigate a meaningful quantity that allows for an intuitive understanding of its convergence properties, we factor out the symmetry breaking parameter $\left(m_{s}-\hat{m}\right)^{2} \propto$ $\left(M_{K}^{2}-M_{\pi}^{2}\right)^{2}$. As an extrapolation now would diverge in the chiral limit due to the appearance of non-analytic quark mass dependence $\propto m_{q}^{-1}, m_{q}^{-1 / 2}$, and in order to obtain a dimen- 

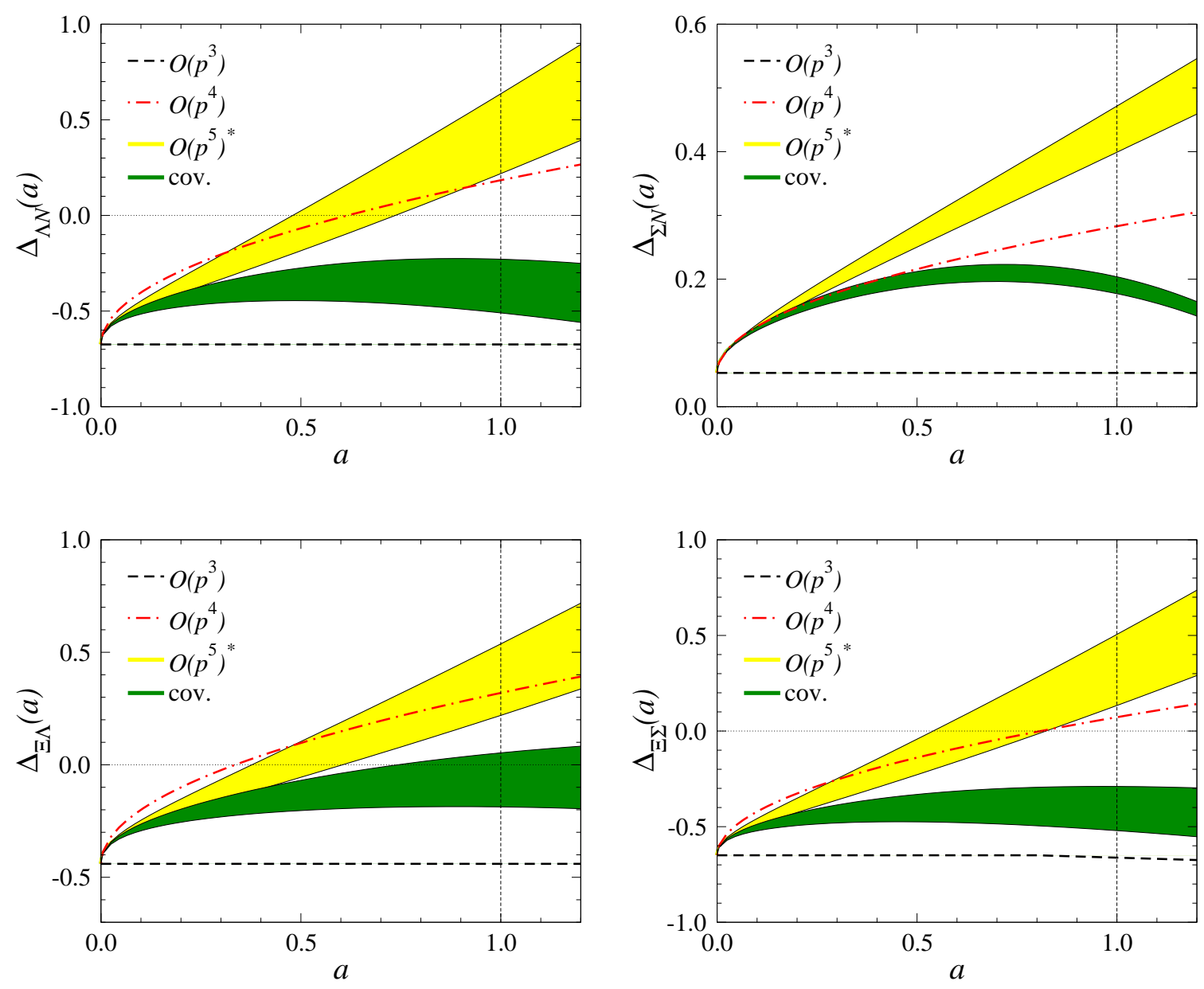

Figure 2: Chiral extrapolations of the hyperon decay form factor for the channels $\Lambda \rightarrow N, \Sigma \rightarrow N$, $\Xi \rightarrow \Lambda$, and $\Xi \rightarrow \Sigma$, according to Eq. (4.7). The yellow (light) band is the region of the fifth order contribution where we vary the scale between the $\rho$ - and the $\Xi$-mass. The green (dark) band is the same variation for the full covariant amplitude.

sionless quantity, we additionally factor out a term proportional to $1 /\left[\left(4 \pi F_{\pi}\right)^{2}\left(M_{\pi}^{2}+M_{K}^{2}\right)\right]$, thus making the quark mass dependence of the leading $\mathcal{O}\left(p^{3}\right)$ symmetry breaking term a simple constant. We introduce a quark-mass scaling parameter $a$ and reparameterize the meson masses according to $M_{\phi} \rightarrow \sqrt{a} M_{\phi}$. In Fig. 2 we plot the reparameterized relative corrections to the vector form factor of all four channels,

$$
\Delta(a)=\left[\frac{a^{2}\left(M_{K}^{2}-M_{\pi}^{2}\right)^{2}}{\left(4 \pi F_{\pi}\right)^{2}\left(M_{\pi}^{2}+M_{K}^{2}\right)}\right]^{-1}\left(\frac{f_{1}(a, t=0)}{g_{V}}-1\right),
$$

order by order. We observe improved convergence for smaller quark masses in all four channels, as expected. As discussed above, the orders $\mathcal{O}\left(p^{5}\right)$ and $C o v$ include a subleading dependence on the renormalization scale, which we vary once more in the region between the $\rho$ - and $\Xi$-mass, producing bands for these orders. It is obvious, though, that there is 

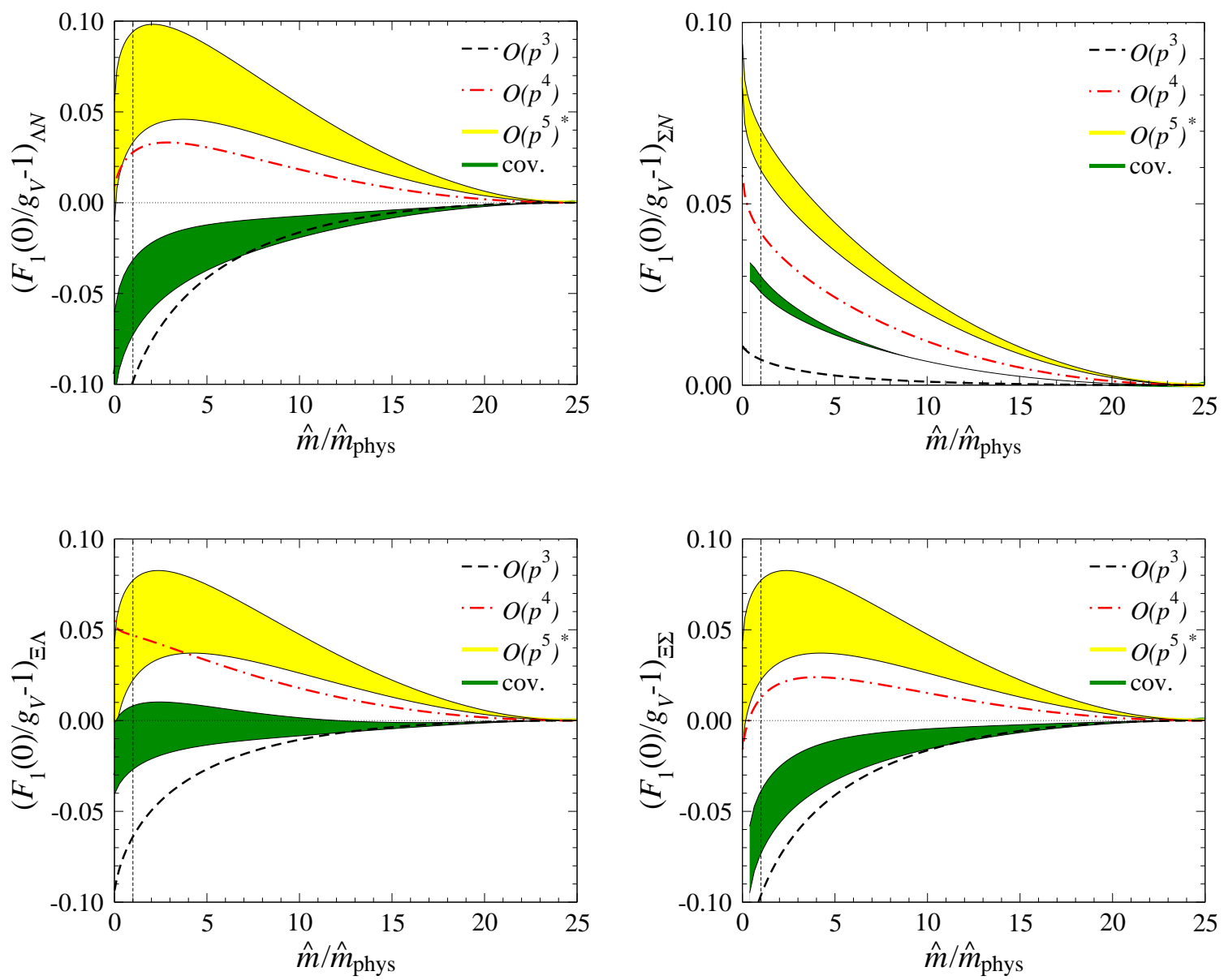

Figure 3: Chiral extrapolations of the hyperon decay form factor for the channels $\Lambda \rightarrow N, \Sigma \rightarrow N$, $\Xi \rightarrow \Lambda$, and $\Xi \rightarrow \Sigma$. We show the dependence on $\hat{m} / \hat{m}_{\text {phys }}$ for fixed $m_{s}$. The yellow (light) band is the region of the fifth order contribution where we vary the scale between the $\rho$ - and the $\Xi$-mass. The green (dark) band is the same variation for the full covariant amplitude.

no good convergence towards the covariant result up to $\mathcal{O}\left(p^{5}\right)$ even for the physical quark masses $(a=1)$, let alone at even higher masses.

As a second application of varying quark masses, and closer in spirit to what is needed for lattice simulations, we increase the (average) light quark mass $\hat{m}$, keeping $m_{s}$ fixed. Obviously the convergence of the chiral series becomes rather more problematic for larger $\hat{m}$ (or, equivalently, larger pion mass). However, in the symmetry limit $\hat{m}=m_{s}$, or $\hat{m} / \hat{m}_{\text {phys }} \approx 25$, the corrections to $f_{1}(0)$ vanish quadratically, as can indeed be seen in all channels and for all chiral orders in Fig. 3. The qualitative picture is very similar for all channels except the $\Sigma \rightarrow N$ one. Numerically, the covariant results are surprisingly close to the leading-order calculation. [Note that the bands for the covariant results for the channels $\Sigma \rightarrow N$ and $\Xi \rightarrow \Sigma$ stop for $\hat{m} / \hat{m}_{\text {phys }} \lesssim 0.35$, where the $\Sigma$ becomes instable against strong decay to $\Lambda \pi$, and $f_{1}(0)$ develops an imaginary part.] 


\subsection{Further comments on the chiral expansion}

Let use briefly return to the results of the chiral expansion for the Dirac form factors at zero momentum transfer displayed in Table 2. First, we note that the corrections taking either the fourth order heavy-baryon results $\left(S u m^{(3)}\right)$ or the full covariant one $(C o v)$ are in the few percent range, with sizeable corrections from $1 / m$ and baryon mass splitting insertions in all cases. The importance of these effects makes the covariant scheme - in which such contributions are resummed to all orders - more reliable than the strict heavybaryon expansion. This is also consistent with the experience made in the calculations of the electromagnetic nucleon [44] and hyperon [31] form factors. It is therefore these covariant results (last entry in Table 2) that should be used in the extraction of $V_{u s}$ from hyperon semileptonic decays.

\section{Weak radii}

For the analysis of ongoing and future high-precision hyperon decay experiments, it will be essential to include the effects of subleading moments in the hyperon decay vector form factors, first of all the weak magnetic moments $f_{2}(0)$, and the leading $t$-dependence of $f_{1}$ as given by the Dirac radii, see Eq. (2.2). As a side remark, we note that the complete $t$ dependence of the one-loop chiral representation of $f_{1}(0)$ displays very little curvature, such that the linear approximation of Eq. (2.2) is in fact rather precise; compare the discussions in Refs. [31, 44].

In this section we analyze the weak Dirac radii of the semileptonic hyperon decays. According to Eq. (3.4), two low-energy constants $d_{51 / 52}$ enter the chiral representations of these radii at third order (and no further constants at $\mathcal{O}\left(p^{4}\right)$ ), which also feature in the electromagnetic Dirac radii of the nucleons $\left\langle r_{1}^{2}\right\rangle_{p / n}[31,45]$. The hyperon decay radii are therefore linked to the latter by low-energy theorems that only contain finite, renormalization-scale-independent loop effects as corrections:

$$
\begin{array}{ll}
\left\langle r_{1}^{2}\right\rangle_{\Lambda N}=\left\langle r_{1}^{2}\right\rangle_{p}+L_{r}(\Lambda N), & \left\langle r_{1}^{2}\right\rangle_{\Sigma N}=\left\langle r_{1}^{2}\right\rangle_{p}+2\left\langle r_{1}^{2}\right\rangle_{n}+L_{r}(\Sigma N), \\
\left\langle r_{1}^{2}\right\rangle_{\Xi \Lambda}=\left\langle r_{1}^{2}\right\rangle_{p}+\left\langle r_{1}^{2}\right\rangle_{n}+L_{r}(\Xi \Lambda), & \left\langle r_{1}^{2}\right\rangle_{\Xi \Sigma}=\left\langle r_{1}^{2}\right\rangle_{p}-\left\langle r_{1}^{2}\right\rangle_{n}+L_{r}(\Xi \Sigma) .
\end{array}
$$

The remaining loop contributions $L_{r}\left(B B^{\prime}\right)$ are non-analytic $\mathrm{SU}(3)$-symmetry breaking effects. A representation according to Eqs. (5.1) holds up to corrections of $\mathcal{O}\left(p^{5}\right)$, where analytic SU(3) breaking due to quark-mass-dependent counterterms is allowed. Furthermore, up to $\mathcal{O}\left(p^{4}\right) f_{1}(0)$ as factored out in Eq. (2.2) can be set to the symmetry limit. As the neutron Dirac radius is strongly suppressed compared to the proton one $\left(\left\langle r_{1}^{2}\right\rangle_{p} \simeq 0.60 \mathrm{fm}^{2}\right.$ vs. $\left\langle r_{1}^{2}\right\rangle_{n} \simeq 0.01 \mathrm{fm}^{2}$, see e.g. Ref. [46]), Eqs. (5.1) suggest hyperon decay radii very close to the proton radius in the $\mathrm{SU}(3)$ symmetry limit.

The analytic expressions for the loop corrections of the low-energy theorems tend to be lengthy, these are collected in Appendix A. In Table 3 we give the numerical values for these as well as for the resulting weak Dirac radii in third and fourth chiral order. The loop contributions at third order tend to be very large, in particular for the channel $\Xi \rightarrow \Sigma$, and corrections at fourth order are similarly sizeable and tend to be opposite in sign, leading 


\begin{tabular}{|c|ccc|rcc|}
\hline Channel & $L_{r}^{(3)}$ & $L_{r}^{(4)}$ & $L_{r}^{(\operatorname{Cov})}$ & $\mathcal{O}\left(p^{3}\right)$ & $\mathcal{O}\left(p^{4}\right)$ & Cov \\
\hline$\Lambda \rightarrow N$ & -0.24 & +0.10 & $-0.16 \pm 0.06$ & 0.35 & 0.70 & $0.44 \pm 0.06$ \\
$\Sigma \rightarrow N$ & +0.31 & +0.10 & $-0.10 \pm 0.05$ & 0.92 & 0.72 & $0.51 \pm 0.05$ \\
$\Xi \rightarrow \Lambda$ & +0.06 & +0.05 & $-0.16 \pm 0.03$ & 0.66 & 0.65 & $0.45 \pm 0.03$ \\
$\Xi \rightarrow \Sigma$ & -0.59 & +0.19 & $-0.12 \pm 0.07$ & -0.01 & 0.77 & $0.46 \pm 0.07$ \\
\hline
\end{tabular}

Table 3: Numerical analysis of the weak Dirac radii squared $\left\langle r_{1}^{2}\right\rangle$ (in units of $\mathrm{fm}^{2}$ ). The columns $L_{r}^{(3)}, L_{r}^{(4)}, L_{r}^{(C o v)}$ show the SU(3)-breaking loop corrections as defined in Eq. (5.1) up to third and fourth order, as well as the full covariant result (the latter with some residual scale dependence). The last three columns present the resulting Dirac radii.

to large cancellations. For a better assessment of the convergence behavior and a more reliable prediction, we again show the covariant loop results that consistently resum all the higher-order $1 / m$ and $\delta m$ corrections. As discussed for the case of $f_{1}(0)$ above, such partial higher-order corrections induce some residual dependence on the renormalization scale, which again we vary around $1 \mathrm{GeV}$, between the masses of the $\rho$ and the $\Xi$. After such resummation, all residual loop effects in the radii are negative and of similar (moderate) size, leading to slightly smaller values for the hyperon decay radii compared to the proton Dirac radius. The corresponding numbers are also displayed in Table 3.

We note that phenomenological model parameterizations $[22,40]$ frequently assume a radius term $\left\langle r_{1}^{2}\right\rangle=12 / M_{V}^{2} \approx 0.50 \mathrm{fm}^{2}$, derived from a dipole parameterization with $M_{V}=0.97 \mathrm{GeV}$ for all hyperon decay modes, agreeing rather well with the values shown in Table 3. This agreement is accidental, however, as the $\mathrm{SU}(3)$ breaking mechanisms are completely different: the dipole mass $M_{V}$ is obtained by scaling the phenomenological dipole mass in the nucleon electromagnetic form factors by $M_{K^{*}} / M_{\rho}$, while in $\chi \mathrm{PT} \mathrm{SU}(3)$ breaking is entirely due to Goldstone boson loop effects up to the order considered here. When resumming the loop contributions in a covariant way, sign and size of the effect agrees with the phenomenological guess.

\section{Weak anomalous magnetic moments}

Comparably to the weak Dirac radii, the weak magnetism form factor at vanishing momentum transfer $f_{2}(0)$ can be related by low-energy theorems to anomalous magnetic moments of the ground state baryon octet. For convenience, we will define weak magnetic moments with a slightly different normalization,

$$
\kappa=\frac{2 m_{N}}{m_{1}} f_{2}(0)
$$

where $m_{1}$ is the mass of the decaying baryon, see Eq. (2.1), such that the magnetic moments $\kappa$ are given in units of nuclear magnetons.

At leading order $\mathcal{O}\left(p^{2}\right)$, the magnetic moments $\kappa_{B B^{\prime}}$ are just given in terms of the low-energy constants $b_{12 / 13}$, see Eq. (3.3), so they can be strictly related to the proton's 


\begin{tabular}{|c|ccc|rrrc|}
\hline Channel & $L_{\kappa}^{(3)}$ & $L_{\kappa}^{(4)}$ & $L_{\kappa}^{(\text {Cov })}$ & $\mathcal{O}\left(p^{2}\right)$ & $\mathcal{O}\left(p^{3}\right)$ & $\mathcal{O}\left(p^{4}\right)$ & Cov \\
\hline$\Lambda \rightarrow N$ & -0.16 & +0.16 & $+0.27 \pm 0.03$ & 1.33 & 1.17 & 1.49 & $1.60 \pm 0.03$ \\
$\Sigma \rightarrow N$ & +0.16 & +0.01 & $+0.03 \pm 0.01$ & -1.59 & -1.43 & -1.58 & $-1.56 \pm 0.01$ \\
$\Xi \rightarrow \Lambda$ & -0.16 & +0.07 & $+0.38 \pm 0.05$ & -0.13 & -0.29 & -0.07 & $0.25 \pm 0.05$ \\
$\Xi \rightarrow \Sigma$ & +0.15 & +0.25 & $-0.02 \pm 0.08$ & 2.45 & 2.60 & 2.71 & $2.43 \pm 0.08$ \\
\hline
\end{tabular}

Table 4: Numerical analysis of the weak anomalous magnetic moments (in nuclear magnetons). The columns $L_{\kappa}^{(3)}, L_{\kappa}^{(4)}, L_{\kappa}^{(\text {Cov })}$ show the SU(3)-breaking loop corrections as defined in Eq. (6.3) up to third and fourth order, as well as the full covariant result (the latter with some residual scale dependence). The last four columns present the resulting anomalous magnetic moments, where the $\mathcal{O}\left(p^{2}\right)$ result is calculated from Eq. (6.3) with vanishing loop corrections.

and neutron's anomalous magnetic moments in analogy to Eq. (5.1),

$$
\begin{array}{rlrl}
\kappa_{\Lambda N} & =g_{V}^{\Lambda N} \kappa_{p}, & \kappa_{\Sigma N} & =g_{V}^{\Sigma N}\left(\kappa_{p}+2 \kappa_{n}\right), \\
\kappa_{\Xi \Lambda}=g_{V}^{\Xi \Lambda}\left(\kappa_{p}+\kappa_{n}\right), & \kappa_{\Xi \Sigma}=g_{V}^{\Xi \Sigma}\left(\kappa_{p}-\kappa_{n}\right) .
\end{array}
$$

Once more $g_{V}$ are the vector couplings defined in Eq. (2.6). No loop corrections can occur at this order.

However, the large number of symmetry-breaking (i.e. quark mass dependent) counterterms contributing to the magnetic moments at fourth order, see Eq. (3.5), prevents a sensible use of a simple low-energy theorem like Eq. (6.2). For a discussion of higherorder corrections to such relations, we therefore resort to relations making use of the well-measured hyperon magnetic moments, whose chiral representations are also known in covariant $\mathrm{B} \chi \mathrm{PT}$ to the appropriate order $[31,45]$. The corresponding low-energy theorems read

$$
\begin{aligned}
\kappa_{\Lambda N} & =g_{V}^{\Lambda N}\left[\frac{1}{6}\left(5 \kappa_{p}+2 \kappa_{n}-6 \kappa_{\Lambda}+\kappa_{\Sigma^{-}}-2 \kappa_{\Xi^{-}}\right)+L_{\kappa}(\Lambda N)\right], \\
\kappa_{\Sigma N} & =g_{V}^{\Sigma N}\left[\frac{1}{2}\left(\kappa_{p}+2 \kappa_{n}-\kappa_{\Sigma^{+}}-2 \kappa_{\Sigma^{-}}\right)+L_{\kappa}(\Sigma N)\right], \\
\kappa_{\Xi \Lambda} & =g_{V}^{\Xi \Lambda}\left[\frac{1}{6}\left(2 \kappa_{p}+6 \kappa_{\Lambda}-\kappa_{\Sigma^{+}}-2 \kappa_{\Xi^{0}}-5 \kappa_{\Xi^{-}}\right)+L_{\kappa}(\Xi \Lambda)\right], \\
\kappa_{\Xi \Sigma} & =g_{V}^{\Xi \Sigma}\left[\frac{1}{2}\left(2 \kappa_{\Sigma^{+}}+\kappa_{\Sigma^{-}}-2 \kappa_{\Xi^{0}}-\kappa_{\Xi^{-}}\right)+L_{\kappa}(\Xi \Sigma)\right] .
\end{aligned}
$$

The remaining loop effects $L_{\kappa}\left(B B^{\prime}\right)$ contain non-analytic symmetry-breaking terms. They are free of fourth-order low-energy constants, and finite up-to-and-including $\mathcal{O}\left(p^{5}\right)$, while our calculation of these corrections is complete up-to-and-including $\mathcal{O}\left(p^{4}\right)$ only. The corresponding formulae for the $L_{\kappa}\left(B B^{\prime}\right)$ are collected in Appendix B.

In Table 4 we present the numerical results, both for the residual loop effects and for the resulting weak anomalous magnetic moments. All numbers are given to second (no loop effects), third, and fourth chiral order, as well as the resummation of $1 / m$ effects in 
the covariant loop representation. The well-known anomalous magnetic moments of the ground state baryons are taken as $\kappa_{p}=1.793, \kappa_{n}=-1.913, \kappa_{\Lambda}=-0.613, \kappa_{\Sigma^{+}}=1.458$, $\kappa_{\Sigma^{-}}=-0.160, \kappa_{\Xi^{0}}=-1.250, \kappa_{\Xi^{-}}=0.349$ [47], all given in nuclear magnetons. Again, we show the effects of the residual higher-order scale dependence in the covariant loop corrections by varying the renormalization scale between the $\rho$ - and the $\Xi$-mass.

Comparing third-, fourth-, and covariant loop order results, we once more observe partially problematic convergence behavior, with the covariant results not always very close to their fourth-order approximations. The total residual loop effects are very small in two channels $(\Sigma \rightarrow N, \Xi \rightarrow \Lambda)$ and more significant in the other two $(\Lambda \rightarrow N, \Xi \rightarrow \Sigma)$.

\section{Conclusions and outlook}

Hyperon semileptonic decays allow for an independent method of extracting $V_{u s}$, provided the hadronic form factors involved are sufficiently well-known from theory. In this article, we have investigated the vector form factors to complete one-loop order in covariant $\mathrm{B} \chi \mathrm{PT}$, with the following findings:

1. The dominant contribution in the vector matrix elements of hyperon decays is due to the Dirac form factor at zero momentum transfer, $f_{1}(0)$. Corrections to the $\mathrm{SU}(3)$ symmetry limit of this quantity are of second order in $m_{s}-\hat{m}$ due to the AdemolloGatto theorem, and are dominated in the chiral power counting by non-analytic loop contributions. We confirm earlier results [15] when performing the heavy-baryon expansion of the covariant loop results, and find problematic convergence behavior of the heavy-baryon series towards the covariant representation.

2. An extrapolation towards smaller quark masses confirms that convergence of the chiral series is restored, but seems problematic at the physical quark mass values. The effects of increasing the average light quark mass $\hat{m}$ at fixed $m_{s}$ are also presented.

3. We have argued drawing upon experience made in earlier calculations of nucleon and hyperon electromagnetic form factors that nevertheless the corrections obtained from the covariant one-loop result should be utilized in the analysis of hyperon semileptonic decays.

4. We have calculated the leading $\mathrm{SU}(3)$ breaking corrections to the radius terms of $f_{1}(t)$. These radii can be related to the electromagnetic Dirac radii of proton and neutron, and corrections are given in terms of parameter-free loop corrections. While the convergence behavior is problematic, the full covariant corrections point at somewhat smaller radii, compared to the proton radius.

5. Furthermore, we have established low-energy theorems that relate the weak anomalous magnetic moments to the magnetic moments of the ground state baryon octet, valid including leading analytic SU(3) breaking effects. The non-analytic loop corrections to these relations are also calculated to complete covariant one-loop order. 
We have not addressed the much more drastic convergence problems found for the inclusion of decuplet effects in the calculation of $f_{1}(0)$ [15]. A reassessment of these effects in covariant $\mathrm{B} \chi \mathrm{PT}[48-51]$ is an important extension of the present work and will be addressed in a future work [52].

\section{Acknowledgements}

We would like to thank Matthias Frink for useful discussions.

\section{A. Loop corrections for the weak Dirac radii}

In this appendix we show analytic expressions for the residual loop contributions to the weak Dirac radii, $L_{r}\left(B B^{\prime}\right)$, as defined in Eq. (5.1), to third and fourth order in the heavybaryon expansion. The third order contributions read

$$
L_{r}^{(3)}\left(B B^{\prime}\right)=\left(\sigma_{B B^{\prime}}^{\eta K}+\rho_{B B^{\prime}}^{\eta K}\right) H_{\eta K}^{(3)}+\left(\sigma_{B B^{\prime}}^{\pi K}+\rho_{B B^{\prime}}^{\pi K}\right) H_{\pi K}^{(3)}+\left(\sigma_{B B^{\prime}}^{K \pi}+\rho_{B B^{\prime}}^{K \pi}\right) H_{K \pi}^{(3)},
$$

with

$$
H_{a b}^{(3)}=\frac{5}{2\left(4 \pi F_{\pi}\right)^{2}}\left\{\frac{M_{a}^{4}\left(3 M_{b}^{2}-M_{a}^{2}\right)}{\left(M_{b}^{2}-M_{a}^{2}\right)^{3}} \log \frac{M_{b}}{M_{a}}-\frac{M_{a}^{2} M_{b}^{2}}{\left(M_{b}^{2}-M_{a}^{2}\right)^{2}}+\frac{5}{12}\right\},
$$

and the coefficients $\sigma_{B B^{\prime}}^{a b}, \rho_{B B^{\prime}}^{a b}$ are given in Table 5. We decompose the additional fourth order corrections into $1 / m$ recoil corrections and $\delta m$ baryon mass splitting corrections according to

$$
L_{r}^{(4)}\left(B B^{\prime}\right)=L_{r}^{(3)}\left(B B^{\prime}\right)+\delta_{r}^{(1 / m)}\left(B B^{\prime}\right)+\delta_{r}^{(\delta m)}\left(B B^{\prime}\right)
$$

The $1 / m$ corrections are

$$
\delta_{r}^{(1 / m)}\left(B B^{\prime}\right)=\rho_{B B^{\prime}}^{\eta K} H_{\eta K}^{(4)}+\rho_{B B^{\prime}}^{\pi K} H_{\pi K}^{(4)}+\rho_{B B^{\prime}}^{K \pi} H_{K \pi}^{(4)},
$$

where

$$
H_{a b}^{(4)}=\frac{\pi}{8\left(4 \pi F_{\pi}\right)^{2}} \frac{M_{a}-M_{b}}{m} \frac{24 M_{a}^{3}+61 M_{a}^{2} M_{b}+44 M_{a} M_{b}^{2}+11 M_{b}^{3}}{\left(M_{a}+M_{b}\right)^{3}} .
$$

The $\delta m$ corrections read

$$
\begin{aligned}
\delta_{r}^{(\delta m)}(\Lambda N)= & (D+F)(D+3 F) H_{K \pi}^{\Lambda \Lambda N}\left(m_{N}\right)-\frac{1}{3}\left(D^{2}-9 F^{2}\right) H_{K \eta}^{\prime \Lambda N}\left(m_{N}\right) \\
& +\frac{2}{3} D(D+3 F) H_{\eta K}^{\prime \Lambda N}\left(m_{\Lambda}\right)+2 D(D-F) H_{\pi K}^{\prime \Lambda N}\left(m_{\Sigma}\right) \\
& +\frac{1}{3}(D+3 F)^{2} H_{\Lambda N}^{(5)}+(D-F)^{2} H_{\Sigma N}^{(5)},
\end{aligned}
$$




\begin{tabular}{|c|cccccc|}
\hline$B \rightarrow B^{\prime}$ & $\sigma_{B B^{\prime}}^{\eta K}$ & $\sigma_{B B^{\prime}}^{\pi K}$ & $\sigma_{B B^{\prime}}^{K \pi}$ & $\rho_{B B^{\prime}}^{\eta K}$ & $\rho_{B B^{\prime}}^{\pi K}$ & $\rho_{B B^{\prime}}^{K \pi}$ \\
\hline$\Lambda \rightarrow N$ & $\frac{3}{5}$ & $\frac{1}{5}$ & $\frac{2}{5}$ & $\frac{1}{3}(D+3 F)^{2}$ & $(D-F)^{2}$ & $2(D+F)^{2}$ \\
$\Sigma \rightarrow N$ & $\frac{3}{5}$ & 1 & $-\frac{2}{5}$ & $3(D-F)^{2}$ & $\frac{7}{3} D^{2}-2 D F+5 F^{2}$ & $-2(D+F)^{2}$ \\
$\Xi \rightarrow \Lambda$ & $\frac{3}{5}$ & $\frac{3}{5}$ & 0 & $\frac{1}{3}(D-3 F)^{2}$ & $3 D^{2}-2 D F+3 F^{2}$ & 0 \\
$\Xi \rightarrow \Sigma$ & $\frac{3}{5}$ & $-\frac{1}{5}$ & $\frac{4}{5}$ & $3(D+F)^{2}$ & $-\frac{11}{3} D^{2}-2 D F-F^{2}$ & $4(D+F)^{2}$ \\
\hline
\end{tabular}

Table 5: Coefficients for Eqs. (A.1), (A.3), (B.1).

$$
\begin{aligned}
\delta_{r}^{(\delta m)}(\Sigma N)= & \left(D^{2}-F^{2}\right) H_{K \pi}^{\prime \Sigma N}\left(m_{N}\right)+(D-F)(D-3 F) H_{K \eta}^{\prime \Sigma N}\left(m_{N}\right) \\
& -\frac{2}{3} D(D+3 F) H_{\pi K}^{\prime \Sigma N}\left(m_{\Lambda}\right)-4(D-F) F H_{\pi K}^{\prime \Sigma N}\left(m_{\Sigma}\right) \\
& +2 D(D-F) H_{\eta K}^{\prime \Sigma N}\left(m_{\Sigma}\right)+\frac{1}{3}(D+3 F)^{2} H_{\Lambda N}^{(5)}+5(D-F)^{2} H_{\Sigma N}^{(5)}, \\
\delta_{r}^{(\delta m)}(\Xi \Lambda)= & \frac{2}{3} D(D-3 F) H_{K \eta}^{\prime \Xi \Lambda}\left(m_{\Lambda}\right)+(D-F)(D-3 F) H_{\pi K}^{\prime \Xi \Lambda}\left(m_{\Xi}\right) \\
& -\frac{1}{3}\left(D^{2}-9 F^{2}\right) H_{\eta K}^{\prime \Xi \Lambda}\left(m_{\Xi}\right)+2 D(D+F) H_{K \pi}^{\prime \Xi \Lambda}\left(m_{\Sigma}\right) \\
& +\frac{1}{3}(D+3 F)^{2} H_{\Lambda N}^{(5)}+3(D-F)^{2} H_{\Sigma N}^{(5)}, \\
\delta_{r}^{(\delta m)}(\Xi \Sigma)= & -\frac{2}{3} D(D-3 F) H_{K \pi}^{\prime \Xi \Sigma}\left(m_{\Lambda}\right)+2 D(D+F) H_{K \eta}^{\prime \Xi \Sigma}\left(m_{\Sigma}\right) \\
& +4(D+F) F H_{K \pi}^{\prime \Xi \Sigma}\left(m_{\Sigma}\right)+(D+F)(D+3 F) H_{\eta K}^{\prime \Xi \Sigma}\left(m_{\Xi}\right) \\
& +\left(D^{2}-F^{2}\right) H_{\pi K}^{\prime \Xi \Sigma}\left(m_{\Xi}\right)+\frac{1}{3}(D+3 F)^{2} H_{\Lambda N}^{(5)}-(D-F)^{2} H_{\Sigma N}^{(5)},
\end{aligned}
$$

with

$$
\begin{aligned}
H_{a b}^{\prime A B}(m) & =\frac{\pi}{\left(4 \pi F_{\pi}\right)^{2}}\left\{\frac{M_{a}^{2}+3 M_{a} M_{b}+M_{b}^{2}}{\left(M_{a}+M_{b}\right)^{3}}\left(m_{A}+m_{B}-2 m\right)-\frac{\left(M_{a}-M_{b}\right)\left(m_{A}-m_{B}\right)}{2\left(M_{a}+M_{b}\right)^{2}}\right\}, \\
H_{A B}^{(5)} & =\frac{5 \pi}{4\left(4 \pi F_{\pi}\right)^{2}} \frac{m_{A}-m_{B}}{M_{K}} .
\end{aligned}
$$

\section{B. Loop corrections for the weak anomalous magnetic moments}

Here we give the analytic residual loop contributions to the weak anomalous magnetic moments, $L_{\kappa}\left(B B^{\prime}\right)$, defined in Eq. (6.3). The third order contributions can be expressed in terms of the same coefficients $\rho_{B B^{\prime}}^{a b}$ given in Table 5 ,

$$
L_{\kappa}^{(3)}\left(B B^{\prime}\right)=\rho_{B B^{\prime}}^{\eta K} H_{\eta K}^{(6)}+\rho_{B B^{\prime}}^{\pi K} H_{\pi K}^{(6)}+\rho_{B B^{\prime}}^{K \pi} H_{K \pi}^{(6)}
$$

with

$$
H_{a b}^{(6)}=\frac{m \pi}{\left(4 \pi F_{\pi}\right)^{2}}\left\{\frac{M_{b}^{2}+M_{a} M_{b}-2 M_{a}^{2}}{3\left(M_{a}+M_{b}\right)}+\frac{M_{a}^{2}-M_{b}^{2}}{2\left(M_{K}+M_{\pi}\right)}\right\} .
$$


The additional fourth order corrections are split into terms scaling with second-order lowenergy constants, $1 / m$ recoil corrections, and $\delta m$ baryon mass splitting corrections,

$$
L_{\kappa}^{(4)}\left(B B^{\prime}\right)=L_{\kappa}^{(3)}\left(B B^{\prime}\right)+\delta_{\kappa}^{(4)}\left(B B^{\prime}\right)+\delta_{\kappa}^{(1 / m)}\left(B B^{\prime}\right)+\delta_{\kappa}^{(\delta m)}\left(B B^{\prime}\right)
$$

These read as follows:

$$
\begin{aligned}
\delta_{\kappa}^{(4)}(\Lambda N)= & m\left(3 b_{5}+b_{6}\right)\left[H_{K \pi}^{(7)}+H_{K \eta}^{(7)}+\frac{8}{3} H_{K \pi}^{(8)}\right]+m b_{7}\left[H_{K \eta}^{(7)}-\frac{4}{3} H_{K \pi}^{(8)}\right] \\
& +\left\{\frac{\kappa_{p}}{4}\left[1-\left(\frac{D}{3}+F\right)^{2}\right]+\frac{4\left(\kappa_{p}+\kappa_{n}\right)}{9}\left(D^{2}-3 F^{2}\right)\right\}\left[H_{K \pi}^{(8)}+3 H_{K \eta}^{(8)}\right], \\
\delta_{\kappa}^{(4)}(\Sigma N)= & 3 m\left(b_{5}-b_{6}\right)\left[H_{K \pi}^{(7)}+H_{K \eta}^{(7)}+\frac{8}{3} H_{K \pi}^{(8)}\right]+m b_{7}\left[H_{K \pi}^{(7)}+4 H_{K \pi}^{(8)}\right] \\
& +\frac{\kappa_{p}+2 \kappa_{n}}{4}\left[1-(D-F)^{2}\right]\left[H_{K \pi}^{(8)}+3 H_{K \eta}^{(8)}\right] \\
\delta_{\kappa}^{(1 / m)}(\Lambda N)= & -\frac{1}{2}\left(3 D^{2}+2 D F+3 F^{2}\right) H_{K \pi}^{(7)}-\frac{1}{9}\left(49 D^{2}+30 D F+57 F^{2}\right) H_{K \pi}^{(8)} \\
& -\frac{1}{6}(D+3 F)^{2} H_{K \eta}^{(7)}+\left(D^{2}-2 D F-7 F^{2}\right) H_{K \eta}^{(8)}, \\
\delta_{\kappa}^{(1 / m)}(\Sigma N)= & -\frac{1}{6}\left(D^{2}-18 D F+9 F^{2}\right) H_{K \pi}^{(7)}+\left(\frac{D^{2}}{3}+10 D F-5 F^{2}\right) H_{K \pi}^{(8)} \\
& -\frac{3}{2}(D-F)^{2}\left[H_{K \eta}^{(7)}+2 H_{K \eta}^{(8)}\right]
\end{aligned}
$$

where

$$
H_{a b}^{(7)}=\frac{1}{\left(4 \pi F_{\pi}\right)^{2}}\left\{\frac{4 M_{b}^{4}}{M_{b}^{2}-M_{a}^{2}} \log \frac{M_{b}}{M_{a}}-M_{a}^{2}-M_{b}^{2}\right\}, \quad H_{a b}^{(8)}=-\frac{M_{b}^{2}}{\left(4 \pi F_{\pi}\right)^{2}} \log \frac{M_{b}}{M_{a}} .
$$

The correction terms $\delta_{\kappa}^{(4)}$ and $\delta_{\kappa}^{(1 / m)}$ for the channels $\Xi \rightarrow \Lambda$ and $\Xi \rightarrow \Sigma$ can be retrieved from the corresponding ones in $\Lambda \rightarrow N$ and $\Sigma \rightarrow N$, respectively, by replacing $F \rightarrow-F$, $b_{6} \rightarrow-b_{6}$, and exchanging $\left(\kappa_{p}+\kappa_{n}\right) \leftrightarrow \kappa_{p}$ throughout. Finally,

$$
\begin{aligned}
\delta_{\kappa}^{(\delta m)}(\Lambda N) & =-(D-F)(D+3 F) H_{K \eta}^{\prime \prime \Lambda N}-\left(D^{2}-6 D F-3 F^{2}\right) H_{K \pi}^{\prime \prime \Lambda N}+2 D(D-F) H^{\prime \prime \prime} \\
& -(D+F)(D-3 F) H_{\Lambda N}^{(9)}-2 D\left[D-F-\left(\frac{7}{9} D-F\right) \log \frac{M_{\pi}}{M_{K}}\right] H_{\Sigma \Lambda}^{(9)}, \\
\delta_{\kappa}^{(\delta m)}(\Sigma N) & =-(D-F)(D+3 F) H_{K \eta}^{\prime \prime \Sigma N}+\left(\frac{5}{3} D^{2}+6 D F-5 F^{2}\right) H_{K \pi}^{\prime \prime \Sigma N}+\frac{2}{3} D(D+3 F) H^{\prime \prime \prime} \\
& +\left(\frac{D^{2}}{3}+2 D F-F^{2}\right) H_{\Lambda N}^{(9)}-\left[\frac{D^{2}}{3}+F^{2}-2 D(D+F) \log \frac{M_{\pi}}{M_{K}}\right] H_{\Sigma \Lambda}^{(9)}, \\
\delta_{\kappa}^{(\delta m)}(\Xi \Lambda) & =(D+F)(D-3 F) H_{K \eta}^{\prime \prime \Xi \Lambda}+\left(D^{2}+6 D F-3 F^{2}\right) H_{K \pi}^{\prime \prime \Xi \Lambda}+2 D(D+F) H^{\prime \prime \prime} \\
& +(D-F)(D+3 F) H_{\Lambda N}^{(9)}-\left[\frac{3}{2}\left(D^{2}+F^{2}\right)+D F-2 D\left(\frac{7}{9} D+F\right) \log \frac{M_{\pi}}{M_{K}}\right] H_{\Sigma \Lambda}^{(9)}, \\
\delta_{\kappa}^{(\delta m)}(\Xi \Sigma) & =(D+F)(D-3 F) H_{K \eta}^{\prime \prime \Xi \Sigma}-\left(\frac{5}{3} D^{2}-6 D F-5 F^{2}\right) H_{K \pi}^{\prime \prime \Xi \Sigma}+\frac{2}{3} D(D-3 F) H^{\prime \prime \prime} \\
& -\left(\frac{D^{2}}{3}-2 D F-F^{2}\right) H_{\Lambda N}^{(9)}-(D-F)\left[\frac{1}{2}(D-F)-2 D \log \frac{M_{\pi}}{M_{K}}\right] H_{\Sigma \Lambda}^{(9)}, \quad(\mathrm{B} .7)
\end{aligned}
$$


with

$$
\begin{aligned}
H_{a b}^{\prime \prime A B} & =\frac{m}{\left(4 \pi F_{\pi}\right)^{2}} \frac{m_{A}-m_{B}}{M_{b}^{2}-M_{a}^{2}}\left\{\frac{1}{4}\left(M_{a}^{2}+M_{b}^{2}\right)-\frac{M_{b}^{4}}{M_{b}^{2}-M_{a}^{2}} \log \frac{M_{b}}{M_{a}}\right\} \\
H^{\prime \prime \prime} & =\frac{m}{\left(4 \pi F_{\pi}\right)^{2}} \frac{M_{\pi}^{2}+M_{K}^{2}}{M_{\pi}^{2}-M_{K}^{2}} \log \frac{M_{\pi}}{M_{K}}\left(m_{\Sigma}-m_{\Lambda}\right), \quad H_{A B}^{(9)}=\frac{m}{\left(4 \pi F_{\pi}\right)^{2}}\left(m_{A}-m_{B}\right) .
\end{aligned}
$$

\section{References}

[1] A. A. Affolder et al. [KTeV E832/E799 Collaboration], "Observation of the decay $\Xi^{0} \rightarrow \Sigma^{+} e^{-} \bar{\nu}_{e}, "$ Phys. Rev. Lett. 82 (1999) 3751.

[2] A. Alavi-Harati et al. [KTeV Collaboration], "First measurement of form factors of the decay $\Xi^{0} \rightarrow \Sigma^{+} e^{-} \bar{\nu}_{e}, "$ Phys. Rev. Lett. 87 (2001) 132001 [arXiv:hep-ex/0105016].

[3] J. R. Batley et al. [NA48/I Collaboration], "Measurement of the branching ratios of the decays $\Xi^{0} \rightarrow \Sigma^{+} e^{-} \bar{\nu}_{e}$ and $\overline{\Xi^{0}} \rightarrow \overline{\Sigma^{+}} e^{+} \nu_{e}$," Phys. Lett. B 645 (2007) 36 [arXiv:hep-ex/0612043].

[4] A. Alavi-Harati et al. [KTeV Collaboration], "Observation of the decay $\Xi^{0} \rightarrow \Sigma^{+} \mu^{-} \bar{\nu}_{\mu}$," Phys. Rev. Lett. 95 (2005) 081801 [arXiv:hep-ex/0504055].

[5] R. Wanke, "New results on $\Xi^{0}$ hyperon decays," arXiv:hep-ex/0702019.

[6] M. Ademollo and R. Gatto, "Nonrenormalization theorem for the strangeness violating vector currents," Phys. Rev. Lett. 13 (1964) 264.

[7] V. Mateu and A. Pich, " $V_{\text {us }}$ determination from hyperon semileptonic decays," JHEP 0510 (2005) 041 [arXiv:hep-ph/0509045].

[8] D. Guadagnoli, V. Lubicz, G. Martinelli, M. Papinutto, S. Simula and G. Villadoro, "Chiral extrapolation of hyperon vector form factors," PoS LAT2005 (2006) 358 [arXiv:hep-lat/0509061].

[9] S. Sasaki and T. Yamazaki, "SU(3) breaking effects in hyperon beta decay from lattice QCD," PoS LAT2006 (2006) 092 [arXiv:hep-lat/0610082].

[10] D. Guadagnoli, V. Lubicz, M. Papinutto and S. Simula, "First lattice QCD study of the $\Sigma^{-} \rightarrow n$ axial and vector form factors with SU(3) breaking corrections," Nucl. Phys. B $76 \mathbf{1}$ (2007) 63 [arXiv:hep-ph/0606181].

[11] H. W. Lin, "| $V_{u s} \mid$ from lattice QCD," arXiv:0707.3647 [hep-ph].

[12] A. Krause, "Baryon matrix elements of the vector current in chiral perturbation theory," Helv. Phys. Acta 63 (1990) 3.

[13] J. Anderson and M. A. Luty, "Chiral corrections to hyperon vector form-factors," Phys. Rev. D 47 (1993) 4975 [arXiv:hep-ph/9301219].

[14] N. Kaiser, "Isospin breaking in neutron $\beta$-decay and $\mathrm{SU}(3)$ violation in semi-leptonic hyperon decays," Phys. Rev. C 64 (2001) 028201 [arXiv:nucl-th/0105043].

[15] G. Villadoro, "Chiral corrections to the hyperon vector form factors," Phys. Rev. D 74 (2006) 014018 [arXiv:hep-ph/0603226].

[16] T. R. Hemmert, B. R. Holstein and J. Kambor, "Heavy baryon chiral perturbation theory with light deltas," J. Phys. G 24 (1998) 1831 [arXiv:hep-ph/9712496]. 
[17] E. E. Jenkins and A. V. Manohar, "Baryon chiral perturbation theory using a heavy fermion Lagrangian," Phys. Lett. B 255 (1991) 558.

[18] E. E. Jenkins and A. V. Manohar, "Chiral corrections to the baryon axial currents," Phys. Lett. B 259 (1991) 353.

[19] N. Fettes and U.-G. Meißner, "Pion nucleon scattering in an effective chiral field theory with explicit spin-3/2 fields," Nucl. Phys. A 679 (2001) 629 [arXiv:hep-ph/0006299].

[20] T. Becher and H. Leutwyler, "Baryon chiral perturbation theory in manifestly Lorentz invariant form," Eur. Phys. J. C 9 (1999) 643 [arXiv:hep-ph/9901384].

[21] V. Bernard, "Chiral perturbation theory and baryon properties," Prog. Part. Nucl. Phys., in press, arXiv:0706.0312 [hep-ph].

[22] A. García, P. Kielanowski and A. Bohm, "The beta decay of hyperons," Lect. Notes Phys. 222 (1985) 1.

[23] S. Weinberg, "Phenomenological Lagrangians," Physica A 96 (1979) 327.

[24] J. Gasser and H. Leutwyler, "Chiral perturbation theory to one loop," Annals Phys. 158 (1984) 142.

[25] J. Gasser and H. Leutwyler, "Chiral perturbation theory: expansions in the mass of the strange quark," Nucl. Phys. B 250 (1985) 465.

[26] V. Bernard and U.-G. Meißner, "Chiral perturbation theory," Ann. Rev. Nucl. Part. Sci. 57 (2007) 33 [arXiv:hep-ph/0611231].

[27] M. Frink and U.-G. Meißner, "On the chiral effective meson-baryon Lagrangian at third order," Eur. Phys. J. A 29 (2006) 255 [arXiv:hep-ph/0609256].

[28] M. Frink and U.-G. Meißner, "The chiral effective meson-baryon Lagrangian at fourth order," in preparation.

[29] J. A. Oller, M. Verbeni and J. Prades, "Meson-baryon effective chiral lagrangians to $\mathcal{O}\left(q^{3}\right)$," JHEP 0609 (2006) 079 [arXiv:hep-ph/0608204]; "Meson-baryon effective chiral Lagrangian at $\mathcal{O}\left(q^{3}\right)$ revisited," arXiv:hep-ph/0701096.

[30] U.-G. Meißner and S. Steininger, "Baryon magnetic moments in chiral perturbation theory," Nucl. Phys. B 499 (1997) 349 [arXiv:hep-ph/9701260].

[31] B. Kubis and U.-G. Meißner, "Baryon form factors in chiral perturbation theory," Eur. Phys. J. C 18 (2001) 747 [arXiv:hep-ph/0010283].

[32] B. Kubis, T. R. Hemmert and U.-G. Meißner, "Baryon form factors," Phys. Lett. B 456 (1999) 240 [arXiv:hep-ph/9903285].

[33] P. Langacker and H. Pagels, "Nonrenormalization theorem in the chiral symmetry limit," Phys. Rev. Lett. 30 (1973) 630.

[34] J. Gasser and H. Leutwyler, "Low-energy expansion of meson form-factors," Nucl. Phys. B 250 (1985) 517.

[35] A. Lacour, "Chiral analysis of hyperon decay vector form factors," Master's thesis, University of Bonn (2007), HISKP-TH-07/02.

[36] J. F. Donoghue, B. R. Holstein and S. W. Klimt, "Kobayashi-Maskawa angles and SU(3) breaking in hyperon beta decay," Phys. Rev. D 35 (1987) 934. 
[37] F. Schlumpf, " $\beta$ decay of hyperons in a relativistic quark model," Phys. Rev. D 51 (1995) 2262 [arXiv:hep-ph/9409272].

[38] R. Flores-Mendieta, " $V_{u s}$ from hyperon semileptonic decays," Phys. Rev. D 70 (2004) 114036 [arXiv:hep-ph/0410171].

[39] H. R. Quinn and J. D. Bjorken, "Renormalization of weak form-factors, and the Cabibbo angle," Phys. Rev. 171 (1968) 1660.

[40] N. Cabibbo, E. C. Swallow and R. Winston, "Semileptonic hyperon decays," Ann. Rev. Nucl. Part. Sci. 53 (2003) 39 [arXiv:hep-ph/0307298].

[41] B. Borasoy and U.-G. Meißner, "Chiral expansion of baryon masses and $\sigma$-terms," Annals Phys. 254 (1997) 192 [arXiv:hep-ph/9607432].

[42] J. F. Donoghue, B. R. Holstein and B. Borasoy, "SU(3) baryon chiral perturbation theory and long distance regularization," Phys. Rev. D 59 (1999) 036002 [arXiv:hep-ph/9804281].

[43] B. Kubis, "Strange form factors and chiral perturbation theory," Eur. Phys. J. A 24S2 (2005) 97 [arXiv:nucl-th/0504004].

[44] B. Kubis and U.-G. Meißner, "Low energy analysis of the nucleon electromagnetic form factors," Nucl. Phys. A 679 (2001) 698 [arXiv:hep-ph/0007056].

[45] B. Kubis, "Strong interactions and electromagnetism in low-energy hadron physics," $\mathrm{PhD}$ thesis, Berichte des FZ Jülich, Jül-4007.

[46] M. A. Belushkin, H. W. Hammer and U.-G. Meißner, "Dispersion analysis of the nucleon form factors including meson continua," Phys. Rev. C 75 (2007) 035202 [arXiv:hep-ph/0608337].

[47] W. M. Yao et al. [Particle Data Group], "Review of particle physics," J. Phys. G 33 (2006) 1.

[48] V. Bernard, T. R. Hemmert and U.-G. Meißner, "Infrared regularization with spin-3/2 fields," Phys. Lett. B 565 (2003) 137 [arXiv:hep-ph/0303198].

[49] V. Bernard, T. R. Hemmert and U.-G. Meißner, "Chiral extrapolations and the covariant small scale expansion," Phys. Lett. B 622 (2005) 141 [arXiv:hep-lat/0503022].

[50] C. Hacker, N. Wies, J. Gegelia and S. Scherer, "Including the $\Delta(1232)$ resonance in baryon chiral perturbation theory," Phys. Rev. C 72 (2005) 055203 [arXiv:hep-ph/0505043].

[51] N. Wies, J. Gegelia and S. Scherer, "Consistency of the $\pi \Delta$ interaction in chiral perturbation theory," Phys. Rev. D 73 (2006) 094012 [arXiv:hep-ph/0602073].

[52] B. Kubis, A. Lacour and U.-G. Meißner, work in progress. 